\title{
Intoxicação experimental pelos frutos de Xanthium cavanillesii (Asteraceae) em ovinos ${ }^{1}$
}

\author{
Alexandre Paulino Loretti ${ }^{2}$, Pedro Soares Bezerra ${ }^{3}$, Marcia Regina Silva Ilha ${ }^{4}$, \\ Severo S. Barros ${ }^{5}$ e Claudio S. L. Barros 6
}

\begin{abstract}
Loretti A.P., Bezerra P.S., Ilha M.R.S., Barros S.S. \& Barros C.S.L. 1999. [Experimental poisoning by the burs of Xanthium cavanillesii (Asteraceae) in sheep.] Intoxicação experimental pelos frutos de Xanthium cavanillesii (Asteraceae) em ovinos. Pesquisa Veterinária Brasileira 19(2):71-78. Depto Patologia Clínica Veterinária, Faculdade de Veterinária, Universidade Federal do Rio Grande do Sul, Av. Bento Gonçalves 9090, Cx. Postal 15094, Porto Alegre, RS 91540-000, Brazil.

The ground burs of Xanthium cavanillesii (Asteraceae) were force fed to 15 adult sheep in single doses or divided in two doses. Nine sheep died. Doses of $2 \mathrm{~g} / \mathrm{kg}$ and above were lethal for the sheep. A single dose of $1,25 \mathrm{~g} / \mathrm{kg}$ and a total of $2,5 \mathrm{~g} / \mathrm{kg}$ divided in two administrations of $1,25 \mathrm{~g} / \mathrm{kg}$ consecutive daily doses did not cause the toxicosis. Clinical signs were observed only in the animals that died and occurred between 5 hours and 20 hours after the beginning of the administration of the burs. The toxicosis had a peracute ( 90 minutes to 3 hours) to acute ( 9 to 13 hours) course. Clinical signs included apathy, anorexia, decreased rate and intensity of ruminal movements, generalized muscle tremors, stiff and incoordinated gait, unwillingness to move, instability, falls and recumbency. Most affected sheep presented seromucous nasal discharge with labored breathing. In terminal stages, there were trismus, drooling of saliva, convulsive seizures, nystagmus, paddling movements, periods of apnea and death. Main necropsy findings included accentuation of the lobular pattern of the liver, focal hemorrhages on the capsular and cut surfaces, distension accompanied by edema and hemorrhages of the gall bladder wall, ascites, hydrotorax, and translucent and gelatinous perirenal edema. The contents of the cecum and proximal loop of the ascending colon were dry, impacted and covered by mucus and streaks of clotted blood; there were disseminated petechiae and suffusions. The main histopathological change consisted of marked coagulative hepatocellular necrosis, which varied from centrilobular to massive, associated with congestion and hemorrhages. In the remaining of the hepatic lobule there was either swelling or vacuolation of the hepatocytes.
\end{abstract}

INDEX TERMS: Poisonous plants, hepatotoxicosis, Xanthium cavanillesii, Asteraceae, diseases of sheep, pathology.

${ }^{1}$ Aceito para publicação em 29 de maio de 1998.

Parte da dissertação de mestrado do primeiro autor. Defendida no Curso de Pós-Graduação em Medicina Veterinária, Área de Concentração em Patologia Veterinária, Centro de Ciências Rurais, Universidade Federal de Santa Maria (UFSM), em 22 de agosto de 1997.

${ }^{2}$ Setor de Patologia Veterinária, Depto Patologia Clínica Veterinária, Faculdade de Veterinária, Universidade Federal do Rio Grande do Sul (UFRGS), Av. Bento Gonçalves 9090, Caixa Postal 15094, Bairro Agronomia, Porto Alegre, RS 91540-000.
3Pós-Graduação em Medicina Veterinária, Área de Concentração em Patologia Veterinária, Centro de Ciências Rurais, UFSM, Santa Maria, RS 97105-900.

${ }^{4}$ Bolsista de Iniciação Científica do CNPq (520121/96-1), Seção de Patologia Veterinária, Depto Patologia, UFSM, Santa Maria, RS 97105-900.

${ }^{5}$ Pesquisador visitante do CNPq, Depto Patologia Animal, Faculdade de Veterinária, Universidade Federal de Pelotas (UFPel), Pelotas, RS 96010-900.

${ }^{6}$ Depto Patologia, UFSM; bolsista do CNPq (350938/91-1). E-mail: cslb@sm.conex.com.br 
RESUMO.- Os frutos moídos de Xanthium cavanillesii foram administrados por via oral, em doses únicas ou fracionadas, a 15 ovinos adultos. Nove ovinos morreram. Doses a partir de $2 \mathrm{~g} / \mathrm{kg}$ em uma única administração foram letais para os ovinos. Doses únicas de $1,25 \mathrm{~g} / \mathrm{kg}$ e doses de $2,5 \mathrm{~g} / \mathrm{kg}$ subdivididas em duas administrações diárias $(1,25 \mathrm{~g} / \mathrm{kg}$ em dois dias consecutivos) não causaram a intoxicação. Sinais clínicos foram observados apenas nos animais que morreram. Iniciavam entre 5 horas e 20 horas após o início da administração dos frutos. A evolução do quadro clínico foi superaguda (90 minutos a 3 horas) ou aguda (9 a 13 horas). Sinais clínicos incluíam apatia, anorexia, hipomotilidade ruminal, tremores musculares generalizados, incoordenação motora, andar rígido, relutância em caminhar, instabilidade, quedas e decúbito. Muitos animais apresentavam corrimento nasal seromucoso que dificultava a respiração. Nas fases terminais havia tiques, sialorréia, convulsões, nistagmo, movimentos de pedalagem e períodos de apnéia seguidos de morte. Os principais achados de necropsia incluíam acentuação do padrão lobular do fígado acompanhada de petéquias distribuídas aleatoriamente na superfície capsular e de corte, distensão da vesícula biliar associada a edema e hemorragias na parede, ascite, hidrotórax, edema gelatinoso e translúcido da região perirrenal, conteúdo do ceco e alça proximal do cólon ascendente ressequido, compactado, recoberto por muco e estrias de sangue coagulado. Havia petéquias e sufusões disseminadas. A principal lesão microscópica consistia em acentuada necrose coagulativa hepatocelular centrolobular ou massiva acompanhada de congestão e hemorragia e vacuolização ou tumefação dos hepatócitos remanescentes.

TERMOS DE INDEXAÇÃO: Plantas tóxicas, hepatotoxicose, Xanthium cavanillesii, Asteraceae, doenças de ovinos, patologia.

\section{INTRODUÇÃO}

Xanthium spp (nomes populares: "carrapicho-de-carneiro" ou "espinho-de-carneiro") são plantas invasoras de culturas anuais com grande capacidade de competição (Kissmann \& Groth 1992). O grau de invasão das lavouras pela planta pode ser tão elevado ao ponto de tornar inevitável a contaminação acidental dos grãos pelos frutos de Xanthium spp, caso a colheita seja realizada mecanicamente.

A intoxicação espontânea por Xanthium spp em animais domésticos ocorre pela ingestão da brotação dicotiledônea quando há carência de forragem, especialmente em áreas que sofrem inundações periódicas (Everist 1974, Kellerman et al. 1988, Mendéz et al. 1994) ou então pela ingestão dos frutos (considerados como a parte mais tóxica da planta) inteiros ou triturados e incluídos acidentalmente na ração de animais confinados (Witte et al. 1990, Driemeier et al. 1998). O quadro clinico-patológico é associado à insuficiência hepática aguda. Os sinais clínicos aparecem poucas horas após a ingestão da planta e incluem tremores musculares (Marsh et al. 1924), incoordenação motora (Martin et al. 1986), andar rígido (Kenny et al. 1950), relutância em caminhar (Stuart et al. 1981), agressividade (Riet-Correa et al. 1984) e, em um estágio mais adiantado da enfermidade, com os animais já em decúbito, hiperestesia (Witte et al. 1990), convulsões (Martin et al. 1986), movimentos de pedalagem (Kingsbury 1964) e cegueira (Martin et al. 1992). A morte ocorre após uma evolução clínica de até 72 horas (Witte et al. 1990). Na necropsia há acentuação do padrão lobular do fígado (Martin et al. 1986, Witte et al. 1990) e edema e hemorragias na parede da vesícula biliar (Riet-Correa et al. 1983, Martin et al. 1992). O achado histopatológico mais significativo é a lesão hepática tóxica clássica de necrose coagulativa centrolobular acompanhada de congestão e hemorragias (Martin et al. 1986). O princípio ativo de Xanthium spp é o glicosideo carboxiatractilosídeo (CAT) (Cole et al. 1980, Stuart et al. 1981, Calanasan et al. 1985).

Em 1996, dois surtos de intoxicação pelos frutos de Xanthium cavanillesii em bovinos foram diagnosticados no Estado do Rio Grande do Sul (Driemeier et al. 1998). Em ambas ocasiões, bovinos confinados estavam recebendo resíduo de lavoura de soja acidentalmente contaminado por grande quantidade dos frutos dessa planta. $O$ uso de grãos na terminação de ovinos torna essa espécie potencialmente sob risco da intoxicação. Dados sobre a intoxicação por Xanthium spp são escassos em ruminantes. Principalmente em ovinos, são superficiais e pouco precisos. A necessidade do conhecimento do quadro clínico, anatomopatológico e ultra-estrutural da intoxicação por Xanthium spp em ovinos tornou oportuna a realização de um estudo experimental com a administração de frutos de $X$. cavanillesii a essa espécie. Descrevem-se as doses tóxicas, o quadro clínico, lesões de necropsia, histopatologia e ultra-estrutura das lesões hepáticas nos ovinos intoxicados experimentalmente.

\section{MATERIAL E MÉTODOS}

Foram utilizados 15 ovinos adultos, fêmeas, mestiços. Eram alimentados com pasto verde e feno de alfafa e recebiam água à vontade. Os frutos de Xanthium cavallinesii Schouw. utilizados no experimento eram procedentes de resíduo de lavoura de soja que havia causado um surto da intoxicação em bovinos em Cachoeira do Sul, RS (Driemeier et al. 1998). Os frutos de X. cavallinesii foram separados manualmente do resíduo e armazenados em temperatura ambiente. Amostras dos componentes do resíduo de soja foram examinadas para pesquisa de micotoxinas. Imediatamente antes da administração aos ovinos, os frutos de $X$. cavallinesii eram moídos finamente (moinho-martelo tipo Willey, malha 20) e misturados em água. A administração era feita por meio de garrafas de vidro em doses únicas ou fracionadas (Quadro 1). Antes da administração, os animais eram submetidos a jejum de 12 a 24 horas. Doses de $2,5 \mathrm{~g} / \mathrm{kg}$ foram subdivididas em duas administrações diárias de $1,25 \mathrm{~g} / \mathrm{kg}$. Exames clínicos foram realizados antes do experimento e duas vezes por dia (pela manhã e ao final da tarde) após a administração dos frutos. Convencionou-se classificar como superagudos os quadro clínicos com evolução de 90 minutos a 3 horas e como agudos os que duraram de 9 a 13 horas.

As necropsias foram realizadas imediatamente após a morte. Órgãos examinados histologicamente incluiram o fígado, coração, linfonodos, baço, pulmão, tubo digestivo, rim, bexiga, adrenal e cérebro. Fragmentos desses órgãos foram processados rotineiramente para histopatologia e corados pela hematoxilina-eosina (HE). Cortes de fígado e rim foram corados pela técnica da hematoxilina fosfotúngstica ácida de Mallory (PTAH) para fibrina. Fragmentos de 
fígado dos ovinos 1 a 3, 7 e 12 foram processados para a microscopia eletrônica. As amostras foram colhidas da face visceral do fígado, fixadas em solução de glutaraldeído a $2 \%$, paraformaldeído a $2 \%$ em tampão cacodilato de sódio, pós-fixadas em tetróxido de ósmio a 1\% no mesmo tampão e incluídas em resina Epon. Cortes ultra-finos foram duplamente contrastados com acetato de uranila e citrato de chumbo.

\section{RESULTADOS}

O delineamento experimental, a evolução do quadro clínico nos animais intoxicados e o desfecho dos experimentos com os frutos de Xanthium cavanillesii realizados em ovinos são apresentados no Quadro 1. Dos quinze ovinos que receberam a planta, nove morreram. A menor dose que causou a morte foi a de $2 \mathrm{~g} / \mathrm{kg}$ em uma única administração (ovino 12) embora o ovino 15 , que recebeu a mesma dose, não tenha adoecido. Os frutos de $X$. cavanillesii causaram a morte dos ovinos 6 e 7 quando administrados em uma única dose de $2,5 \mathrm{~g} / \mathrm{kg}$, mas essa mesma dose total não provocou a intoxicação de outros dois ovinos (ovinos 10 e 11) quando fornecida em duas administrações diárias $(1,25 \mathrm{~g} / \mathrm{kg}$ por dia em dois dias consecutivos). Os sinais clínicos foram observados apenas nos animais que morreram. Iniciavam entre 5 horas e 20 horas após o começo da administração dos frutos. O quadro clínico foi superagudo ou agudo com evolução oscilando entre 90 minutos e 13 horas.

Quadro 1. Evolução do quadro clínico e desfecho. Intoxicação experimental pelos frutos de Xanthium cavanillesii (Asteraceae) em ovinos

\begin{tabular}{rccccc}
\hline Ovino & $\begin{array}{r}\text { Peso } \\
(\mathrm{kg})\end{array}$ & $\begin{array}{c}\text { Dose } \\
(\mathrm{g} / \mathrm{kg})\end{array}$ & $\begin{array}{c}\text { Início dos sinais } \\
\text { clínicos }^{\mathrm{a}}\end{array}$ & Evolução & Desfecho \\
\hline 1 & 32 & 20 & $5 \mathrm{~h}$ & $3 \mathrm{~h}$ & $\mathrm{M}^{\mathrm{c}}$ \\
2 & 29 & 15 & $6 \mathrm{~h} 30 \mathrm{~min}$ & $2 \mathrm{~h}$ & $\mathrm{M}$ \\
3 & 26 & 10 & $7 \mathrm{~h}$ & $3 \mathrm{~h}$ & $\mathrm{M}$ \\
13 & 31 & 10 & $6 \mathrm{~h}$ & $2 \mathrm{~h}$ & $\mathrm{M}$ \\
4 & 28 & 7,5 & $5 \mathrm{~h} 30 \mathrm{~min}$ & $2 \mathrm{~h}$ & $\mathrm{M}$ \\
5 & 32 & 5,0 & $6 \mathrm{~h} 30 \mathrm{~min}$ & $1 \mathrm{~h} 30 \mathrm{~min}$ & $\mathrm{M}$ \\
6 & 28 & 2,5 & $11 \mathrm{~h} 30 \mathrm{~min}$ & $8 \mathrm{~h} 30 \mathrm{~min}$ & $\mathrm{M}$ \\
7 & 26 & 2,5 & $12 \mathrm{~h} 30 \mathrm{~min}$ & $13 \mathrm{~h}$ & $\mathrm{M}$ \\
12 & 29 & 2,0 & $20 \mathrm{~h}$ & $13 \mathrm{~h}$ & $\mathrm{M}$ \\
15 & 31 & 2,0 & - & - & $\mathrm{NA}$ \\
8 & 33 & 1,25 & - & - & NA \\
9 & 36 & 1,25 & - & - & NA \\
14 & 38 & 1,25 & - & - & NA \\
$10^{\mathrm{b}}$ & 37 & 1,25 & - & - & NA \\
$11^{\mathrm{b}}$ & 32 & 1,25 & - & & NA \\
\hline
\end{tabular}

aApós o início da administração.

besses ovinos receberam duas administrações de $1,25 \mathrm{~g} / \mathrm{kg}(1,25 \mathrm{~g} / \mathrm{kg} / \mathrm{dia}$ em dois dias consecutivos). Todos os outros receberam administrações únicas. ${ }^{\mathrm{c}} \mathrm{M}=$ morte.

${ }^{\mathrm{N} A}=$ não adoeceu.

\section{Sinais clínicos}

De um modo geral, o quadro clínico foi bastante uniforme e caracterizou-se principalmente por sinais neuromusculares e respiratórios. Foram observados inicialmente apatia, anorexia e hipomotilidade ruminal seguida de atonia do rúmen. Os animais ficavam acentuadamente prostrados, permanecendo em estação, imóveis e com as orelhas caídas, totalmente alheios ao ambiente (sonolentos), com o olhar fixo e movimentos mastigatórios vazios. Apresentavam ptose palpebral, e mantinham a cabeça baixa, próxima ao solo. Havia abundante corrimento seromucoso espesso pelas narinas que aglutinava os pêlos circunjacentes. Tremores musculares foram um sinal clínico constante. Apareciam já no início do quadro clínico como tremores discretos na cabeça e se intensificavam progressivamente acometendo também os músculos dos membros até tornarem-se generalizados. Quando os ovinos eram exercitados, os tremores acentuavam-se. Alterações na marcha foram observadas com frequência. Caracterizavam-se por acentuada incoordenação motora, andar cambaleante, relutância em caminhar, travando os membros, ou andar rígido, com passos curtos, vacilantes e lentos. Os ovinos afetados caminhavam somente quando forçados e logo paravam. Apresentavam acentuado desequilíbrio, balançando o corpo de um lado para o outro e para a frente ou então permaneciam com os membros posteriores cruzados. Devido à grande instabilidade, mantinham os membros lateralmente afastados e a cabeça baixa próxima ao solo, assumindo uma "posição-de-cavalete" (Fig. 1). Demonstravam sinais de fraqueza manifestados por dificuldade em manter-se em estação e erguer a cabeça, apoio contra as paredes da baia e quedas. Apresentavam respiração acelerada, abdominal, ou laboriosa, com movimentos respiratórios profundos e prolongados. Um animal (ovino 7) caminhava lentamente em círculos, sempre com a cabeça baixa. Tiques ocorriam nas comissuras labiais, no focinho e nas pálpebras. Os animais permaneciam, às vezes, com os membros posteriores unidos, parcialmente flexionados. Em algumas situações, flexionavam os anteriores, ficando de joelhos, porém o trem posterior permanecia erguido. Finalmente, entravam em decúbito esterno-abdominal. Esticavam o pescoço, apoiando o mento ou o focinho no chão, mantendo os posteriores flexionados junto ao corpo. Dois animais (ovinos 2 e 7), quando em decúbito, ficavam com os membros anteriores estendidos para frente ou flexionados e os posteriores estendidos para trás (Fig. 2). Faziam diversas tentativas, sem êxito, em levantar. Posteriormente, assumiam o decúbito lateral. Quando em decúbito, os ovinos demonstravam dor por gemidos e berros. Havia convulsões, caracterizadas por contrações espásticas e repetidas dos membros e do pescoço. Hiperestesia, movimentos de pedalagem intensos, algumas vezes com a cabeça voltada para trás, e sialorréia profusa com acúmulo de espuma branca na boca foram também observados. Em cinco animais (ovinos 3, 4, 6, 7 e 12) houve elevação da temperatura corporal. Em três ovinos foi verificada a presença de pequena quantidade de sangue vivo no reto (ovinos 3,7 e 12). A quantidade excessiva de secreção seromucosa na cavidade nasal dificultava a respiração dos animais, principalmente a expiração que era ruidosa. Em uma fase terminal, os ovinos apresentavam redução da frequência respiratória, com respiração superficial e dispnéica, esticando o pescoço e respirando pela boca. Os períodos de apnéia aumentavam progressivamente até cerca de 1 minuto. Havia ausência da sensibilidade corneal, 

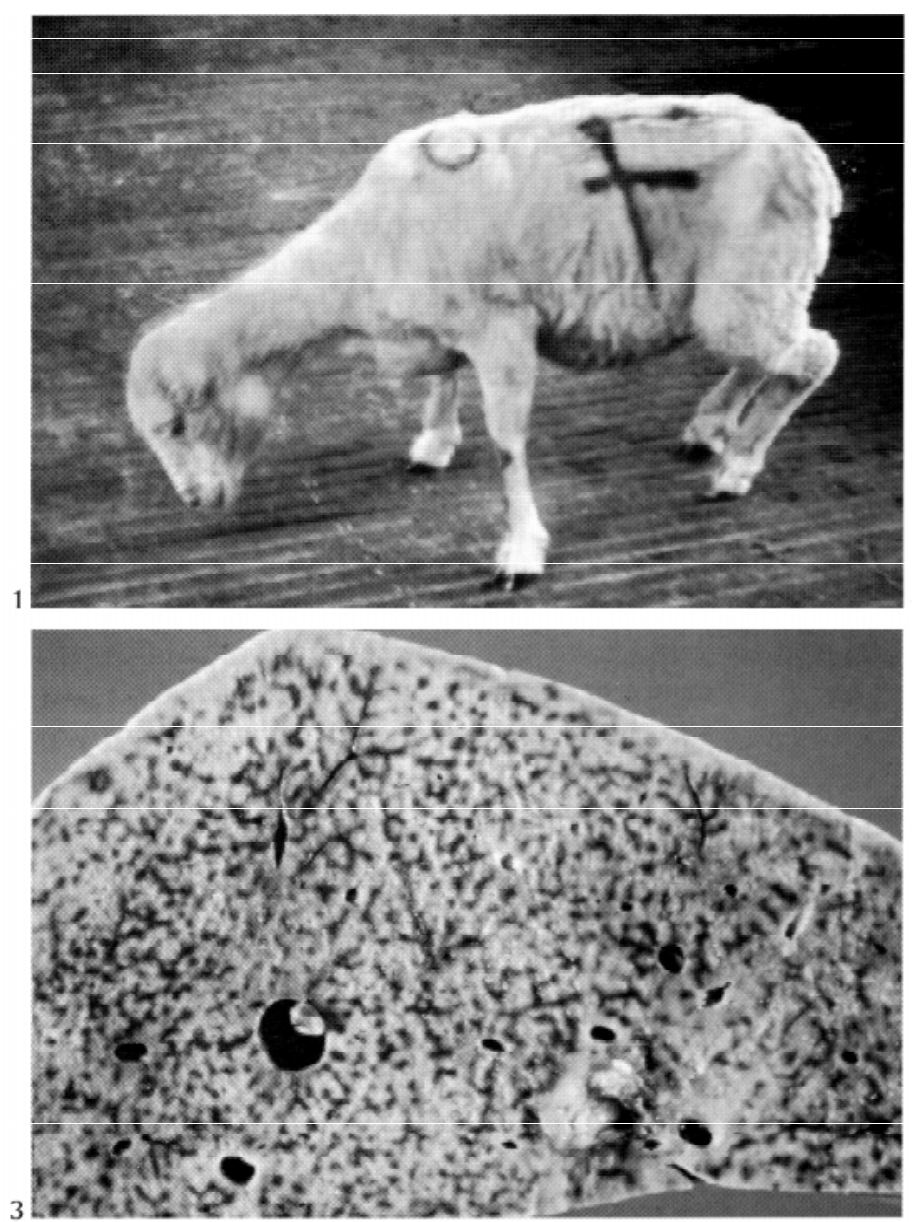

Fig. 1. Acentuada instabilidade e dificuldade de permanecer em estação. Os membros posteriores estão unidos e parcialmente flexionados, os anteriores lateralmente afastados e a cabeça baixa ("posição-de cavalete"). Intoxicação experimental pelos frutos de Xanthium cavanillesii (Asteraceae) em ovinos (Ov. 7).

Fig. 3. Superfície de corte do fígado com acentuação do padrão lobular caracterizada por áreas vermelho-escuras e deprimidas alternadas por áreas claras (aspecto de noz-moscada). Espécimen fixado em formol. Intoxicação experimental pelos frutos de Xanthium cavanillesii (Asteraceae) em ovinos (Ov. 12).

midríase, olhar arregalado e voltado para cima e nistagmo vertical, caracterizando um quadro comatoso de curta duração seguido de morte.

\section{Achados de necropsia}

A principal lesão macroscópica foi observada no fígado, ocorreu nos nove ovinos necropsiados e caracterizava-se por acentuação do padrão lobular na superfície capsular e de corte (Fig. 3). A intensidade dessa lesão variou de leve (ovinos $4 \mathrm{e}$ 6), moderada (ovino 13) ou acentuada (ovinos 1 a 3, 5, 7 e 12). Na superfície de corte apareciam áreas vermelho-escuras e deprimidas alternadas com áreas claras alaranjadas, conferindo ao órgão um aspecto de noz-moscada (Fig. 3). Em um animal (ovino 13), o figado estava levemente aumentado
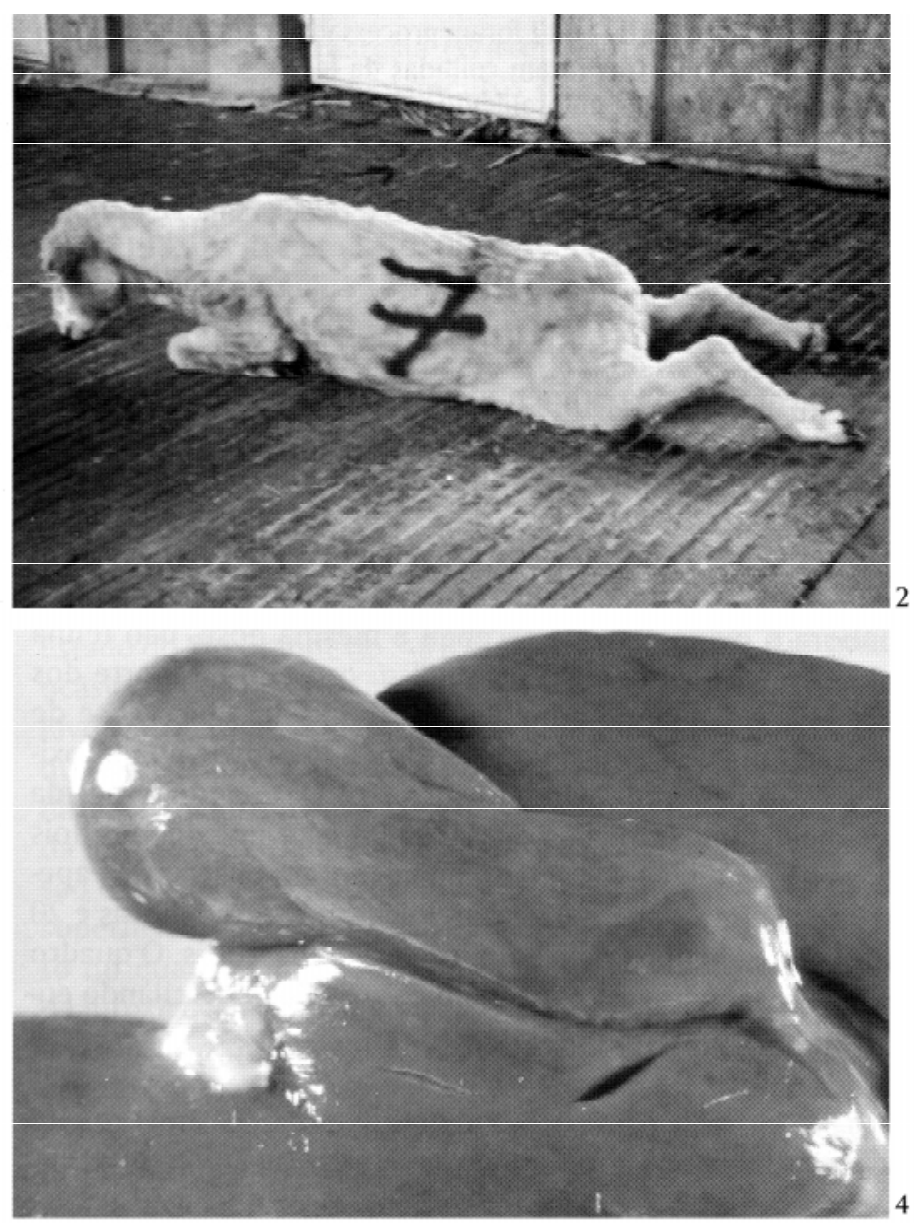

Fig. 2. Decúbito esterno-abdominal. Observe os membros posteriores estendidos para trás, os anteriores flexionados junto ao corpo e o focinho apoiado no chão. Intoxicação experimental pelos frutos de Xanthium cavanillesii (Asteraceae) em ovinos (Ov. 7).

Fig. 4. Distensão da vesícula biliar associada a edema e sufusões na parede. Intoxicação experimental pelos frutos de Xanthium cavanillesii (Asteraceae) em ovinos (Ov. 3).

de volume, vermelho-escuro, com os bordos arredondados e acentuadamente congesto. A acentuação do padrão lobular era, em parte, obscurecida pela congestão. Na superfície de corte do fígado do ovino 1, havia extensas áreas vermelhoescuras, irregulares, focalmente extensas, mais evidentes após a fixação. Nos ovinos 1, 3, 5, 6 e 12 havia numerosas petéquias distribuídas aleatoriamente na superfície capsular e de corte. Em dois casos (ovinos 3 e 5), a superfície hepática estava recoberta por filamentos de fibrina. Com exceção do ovino 1, em todos os animais necropsiados foi observada distensão da vesícula biliar. Nos ovinos 2, 3 e 7 havia também graus variáveis de edema na parede da vesícula biliar (Fig. 4). Sufusões foram observadas na parede da vesícula biliar dos ovinos 2, 3 e 13 (Fig. 4). Graus variáveis de ascite ocorreram 

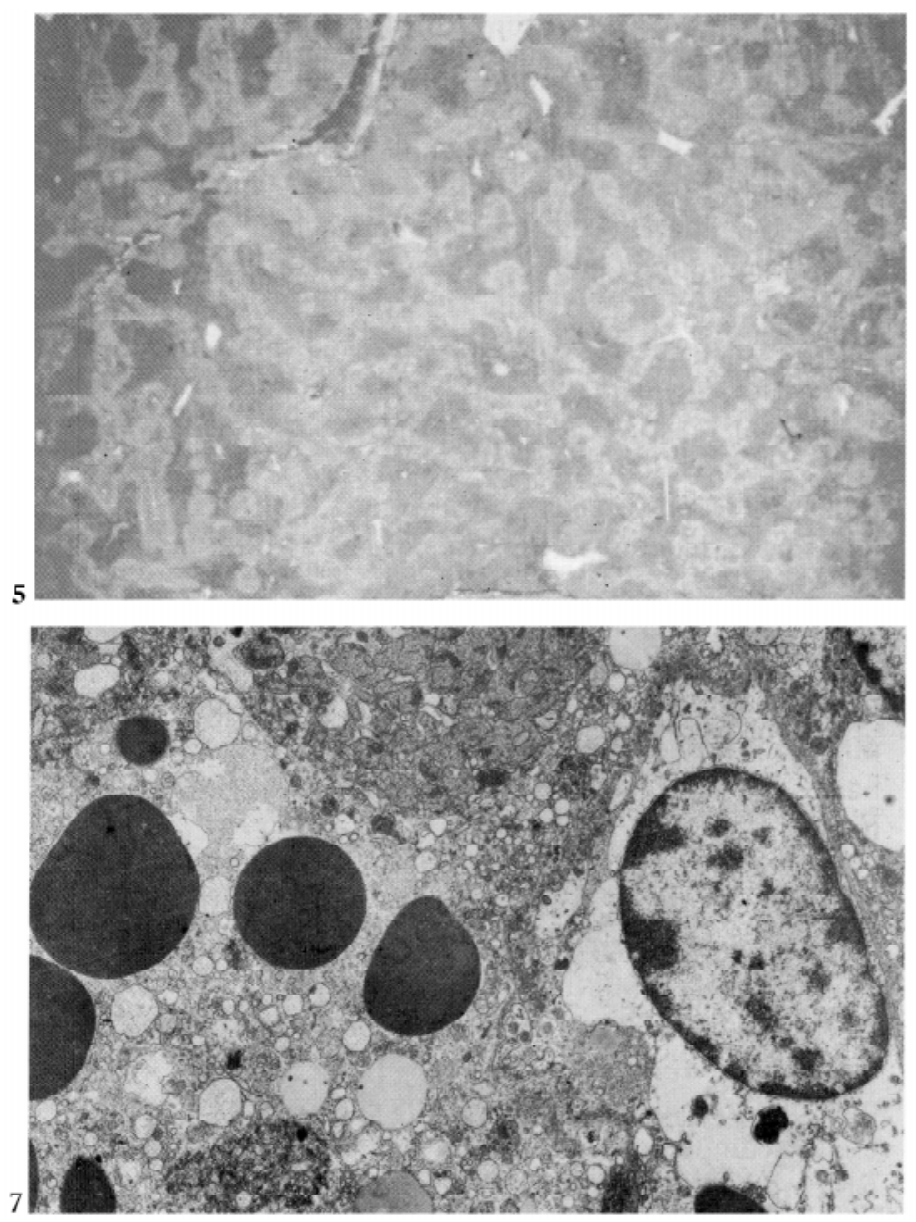

Fig. 5. Fígado com áreas de necrose coagulativa fortemente eosinofílicas e confluentes (em ponte). Intoxicação experimental pelos frutos de Xanthium cavanillesii (Asteraceae) em ovinos (Ov. 3). HE, obj. 2,5.

Fig. 7. Tumefação da célula endotelial (à direita) e ruptura de um segmento da parede do capilar sinusóide do fígado. Dilatação do espaço de Disse (à esquerda) que contém restos celulares oriundos de hepatócitos necróticos. Intoxicação experimental pelos frutos de Xanthium cavanillesii (Asteraceae) em ovinos (Ov. 3). 7.200x.

consistentemente, exceto nos ovinos 6 e 12. O aspecto da efusão cavitária variava de límpido e claro a opaco e viscoso. Em 2 casos (ovinos 3 e 7) havia filamentos e flocos de fibrina misturados ao líquido ascítico. Esse líquido, com exceção do ovino 1, coagulava quando exposto ao ar. Hidrotórax leve foi observado em 3 animais (ovinos 5, 7 e 13). Caracterizava-se pela presença de líquido citrino, límpido e claro que não coagulava quando exposto ao ar. Edema gelatinoso e translúcido moderado na região perirrenal foi observado nos ovinos 2 e 13. O conteúdo do ceco e da alça proximal do cólon ascendente estava ressequido, compactado e recoberto por muco e estrias de sangue coagulado (ovinos 3 a 7 e 12). Petéquias e sufusões foram frequentemente observadas em diversos órgãos.
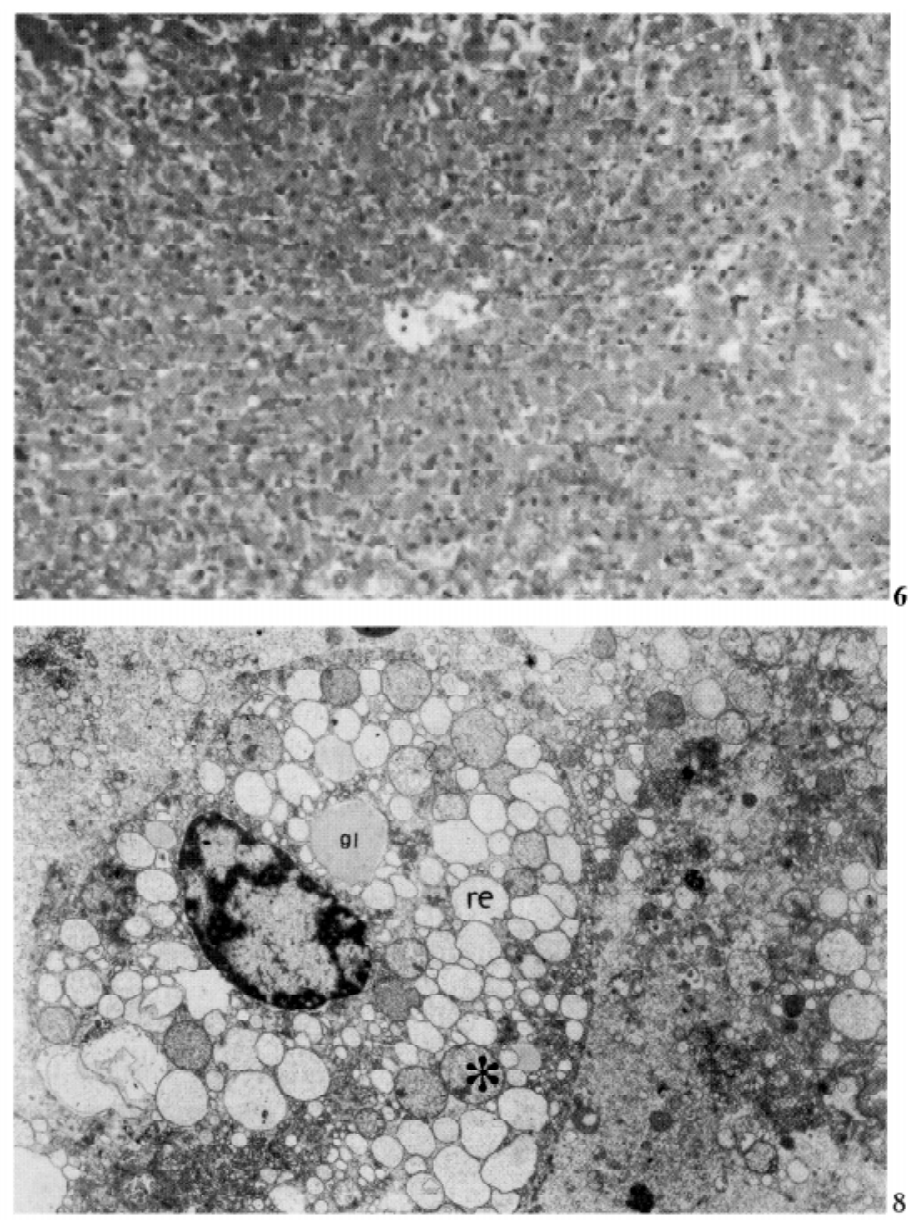

Fig. 6. Fígado com necrose coagulativa hemorrágica. A lesão atinge quase a totalidade do lóbulo hepático, preservando apenas de dois a três fileiras de hepatócitos (necrose massiva). Intoxicação experimental pelos frutos de Xanthium cavanillesii (Asteraceae) em ovinos (Ov. 1). HE, obj. 10.

Fig. 8. Hepatócito com acentuada dilatação do retículo endoplasmático (re), tumefação mitocondrial de grande amplitude, gotículas de lípídio (gl), densidades matricárias (*) e picnose nuclear. Ovino 3 na intoxicação experimental pelos frutos de Xanthium cavanillesii. 30.000x.

Achados histopatológicos e ultra-estruturais

A principal alteração histopatológica, encontrada em todos os ovinos necropsiados, consistia em acentuada necrose coagulativa hepatocelular centrolobular (ovinos 2, 3, 5, 7, 12 e 13) (Fig. 5) ou massiva (ovinos 1, 4 e 6) (Fig. 6). Essa última distribuição caracterizava-se por necrose de quase todo o lóbulo hepático, permanecendo viáveis apenas duas ou três fileiras de hepatócitos na periferia. A lesão centrolobular atingia cerca de dois terços do lóbulo a partir da veia central. As lesões necróticas eram confluentes (em ponte), afetando as zonas 3 e 2 (ácino hepático de Rappaport) e associadas à congestão e hemorragia centrolobulares (ovinos 2 a 7, 12 e 13) ou distribuídas irregularmente (ovino 1). Esférulas arredondadas, eosinofílicas, homogêneas, negativas na técnica da 
PTAH, localizadas no citoplasma de hepatócitos, nos espaços de Disse e na luz sinusoidal, ocorreram com frequência. Pequena quantidade de neutrófilos e de células mononucleares preenchia a luz de veias centrolobulares e sinusóides, especialmente daqueles localizados ao redor da veia central. Foi observada também tumefação das células endoteliais dos sinusóides, ativação das células de Kupffer e edema dos espaços de Disse. Ultra-estruturalmente confirmou-se a dilatação dos espaços de Disse que estavam preenchidos por restos celulares oriundos de hepatócitos necróticos e por neutrófilos. Adicionalmente, havia lesão acentuada das células endoteliais dos sinusóides, ruptura de segmentos da parede desses capilares com o surgimento de áreas de descontinuidade acompanhadas de agregação plaquetária. As esférulas eosinofílicas observadas na microscopia óptica correspondiam, na microscopia eletrônica, a porções do citoplasma de hepatócitos sequestrados intra-celularmente ou desprendidos para os espaços de Disse ou então para a luz dos sinusóides (Fig. 7). Hepatócitos da região periportal constantemente apresentavam vários graus de alterações regressivas caracterizadas por tumefação e vacuolização do citoplasma. $\mathrm{O}$ citoplasma dessas células tinha aspecto granular ou vesicular, núcleo aumentado cerca de duas vezes o tamanho normal, vesicular e com marginação da cromatina. Ocasionalmente, observava-se, nessa região, lise de hepatócitos, intimamente relacionada à marcada tumefação. Vacuolização dos hepatócitos foi observada com frequência e variava de moderada a acentuada. Os vacúolos intracitoplasmáticos eram bem delimitados, pequenos e numerosos ou grandes e únicos. Na microscopia eletrônica observava-se, nos hepatócitos, acentuada dilatação ou agregação das cisternas do retículo endoplasmático. Nas mitocôndrias havia tumefação de grande amplitude, densidades matricárias e calcificação. Gotas lipídicas envoltas por membranas, desaparecimento dos grânulos de glicogênio e picnose nuclear foram também observadas no citoplasma de hepatócitos (Fig. 8).

Alterações regressivas discretas ou moderadas nas células epiteliais dos túbulos contorcidos proximais e proteinose tubular moderada foram as principais alterações histológicas observadas nos rins.

Macrófagos com citoplasma espumoso foram observados em linfonodos de todos os ovinos necropsiados com exceção do ovino 13.

O exame toxicológico do resíduo de lavoura de soja não revelou micotoxinas.

\section{DISCUSSÃO}

Nos experimentos efetuados neste estudo, constatou-se que os frutos moídos de Xanthium cavanillesii são tóxicos para ovinos. A administração de doses únicas produziu um quadro tóxico superagudo ou agudo fatal nos ovinos que ingeriram quantidades de 2 a $20 \mathrm{~g} / \mathrm{kg}$. Comparando esses dados com os dos estudos experimentais com a brotação dicotiledônea de Xanthium sp em ovinos (Méndez et al. 1994, Santos 1997), verifica-se que a toxidez dos frutos de $X$. cavanillesii para esta espécie é dez vezes maior do que a da brotação dicotiledônea. Achados semelhantes são descritos na intoxi- cação de ruminantes e suínos por Xanthium spp (Horváth \& Modor 1970).

$O$ princípio tóxico dos frutos de $X$. cavanillesii demonstrou-se primariamente hepatotóxico. $O$ quadro clínicopatológico reproduzido é consistente com o de uma insuficiência hepática aguda em consequência de necrose aguda acentuada do parênquima hepático. Os sinais clínicos, os dados de necropsia e as alterações microscópicas observados são semelhantes aos descritos na intoxicação espontânea e experimental por Xanthium spp em bovinos (Martin et al. 1986, Witte et al. 1990, Martin et al. 1992, Méndez et al. 1994, Driemeier et al. 1998) e suínos (Kenny et al. 1950, Stuart et al. 1981, Oelkers \& Oehme 1982). O ovino pode, portanto, ser utilizado como modelo experimental para os estudos sobre a intoxicação por Xanthium spp em ruminantes.

A evolução clínica da intoxicação experimental foi dosedependente. Animais que receberam doses mais altas (entre 5 e $20 \mathrm{~g} / \mathrm{kg}$ ) tiveram evolução clínica superaguda (de $90 \mathrm{mi}$ nutos a 3 horas) enquanto os que receberam as doses mais baixas ( 2 e 2,5 g/kg) tiveram evolução clínica aguda (de 9 a 13 horas).

Efeito acumulativo é relatado para algumas plantas que causam hepatotoxicoses agudas em ruminantes, p.ex. Cestrum corymbosum var. hirsutum (Gava et al. 1991) e Sessea brasiliensis (Chaulet et al. 1990). Tal efeito não foi observado nesse experimento a partir da administração da dose letal de $2,5 \mathrm{~g} / \mathrm{kg}$, fracionada em duas doses diárias $(1,25 \mathrm{~g} / \mathrm{kg} / \mathrm{dia})$, a dois ovinos, que não manifestaram sinais clínicos de intoxicação. $O$ efeito acumulativo também não foi observado em experimentos com Xanthium spp em bovinos, ovinos e suínos (Stuart et al. 1981, Santos 1997).

Não houve correlação consistente entre a dose e a intensidade da lesão hepática. Lesões acentuadas que afetavam quase a totalidade do lóbulo hepático, preservando apenas uma estreita faixa de hepatócitos (necrose massiva ou panlobular), foram observadas nos ovinos que receberam 20 $\mathrm{g} / \mathrm{kg}, 7,5 \mathrm{~g} / \mathrm{kg}$ e $2,5 \mathrm{~g} / \mathrm{kg}$ (ovino 6) enquanto lesões que afetavam cerca de dois terços do lóbulo foram vistas nos ovinos que receberam $15 \mathrm{~g} / \mathrm{kg}, 10 \mathrm{~g} / \mathrm{kg}, 5 \mathrm{~g} / \mathrm{kg}, 2,5 \mathrm{~g} / \mathrm{kg}$ (ovino 7) e 2 $\mathrm{g} / \mathrm{kg}$.

Várias plantas e substâncias primariamente hepatotóxicas são também nefrotóxicas. A intensidade da lesão tubular muitas vezes está relacionada ao tempo de exposição à toxina e evolução clínica do quadro da intoxicação (Jones et al. 1997). Lesões renais também são descritas na intoxicação por Xanthium spp (Martin et al. 1986, Witte et al. 1990). As alterações histológicas encontradas nos rins dos ovinos deste estudo foram, no entanto, bastante moderadas.

Lesões microscópicas descritas no sistema nervoso central na intoxicação por Xanthium spp incluem cromatólise, astrogliose, degeneração neuronal isquêmica, status spongiosus, congestão e hemorragias (Witte et al. 1990, Santos 1997). Distúrbios neuromusculares foram observados nos ovinos desse experimento e são descritos frequentemente nas hepatotoxicoses agudas em ovinos causadas pela ingestão de plantas p.ex. Vernonia mollissima (Gava et al. 1987), Sessea brasiliensis (Chaulet et al. 1990), Wedelia glauca (Collazo \& Riet- 
Correa 1996), Microcystis aeruginosa (Jackson et al. 1984) e larvas de insetos como Perreya flavipes (Dutra et al. 1997). No entanto, não foram observadas quaisquer alterações microscópicas no encéfalo dos ovinos desse experimento. É possível que devido à evolução clínica curta, não tenha havido tempo suficiente para o desenvolvimento de lesões perceptíveis na microscópia óptica. Em muitas intoxicações por plantas hepatotóxicas em ruminantes não há correlação entre a intensidade dos sinais clínicos de distúrbios nervosos e o grau de status spongiosus no sistema nervoso central. Alternativamente, os distúrbios neuromusculares poderiam ser atribuídos à hipoglicemia que é uma alteração bioquímica observada com frequência na intoxicação por Xanthium spp (Stuart et al. 1981, Martin et al. 1992).

As alterações hepáticas macro e microscópicas observadas nos ovinos desse experimento são as alterações clássicas de lesão hepática aguda de origem tóxica. São descritas na intoxicação por Xanthium spp (Martin et al. 1986, Witte et al. 1990, Méndez et al. 1994, Driemeier et al. 1998) e em outras hepatotoxicoses agudas em ruminantes (Kingsbury 1964).

Ascite e hidrotórax foram achados de necropsia frequentes nos ovinos deste experimento. Efusões cavitárias são comumente relatadas na intoxicação por Xanthium spp em várias espécies (Everist 1974, Stuart et al. 1981, Witte et al. 1990, Martin et al. 1992, Santos 1997). Ascite e hidrotórax são descritos em outras hepatotoxicoses agudas em ovinos como as causadas pela ingestão de Cestrum laevigatum (Van Der Lugt et al. 1992), M. aeruginosa (Jackson et al. 1984), Wedelia glauca (Collazo \& Riet-Correa 1996) e Perreya flavipes (Dutra et al. 1997). É provável que o aumento da permeabilidade vascular tenha sido o mecanismo responsável pelos edemas cavitários observados nos ovinos deste estudo. Em experimento em suínos com $X$. strumarium, foram observadas efusões cavitárias ricas em albumina, globulinas séricas e fibrina e é sugerido que esses derrames estejam relacionados ao aumento de permeabilidade vascular (Stuart et al. 1981). Em outras hepatotoxicoses agudas em ovinos, descreve-se que esse transudato coagula com a exposição ao ar, o que fortalece a hipótese de que haja lesão endotelial (Jackson et al. 1984).

Coagulação intravascular disseminada associada à presença de trombos de fibrina nos sinusóides hepáticos tem sido descrita nas hepatotoxicoses agudas (Verstraete et al. 1974). Nos ovinos desse estudo, essa alteração não pôde ser confirmada pois não se observou fibrina polimerizada na luz dos capilares hepáticos e renais.

As alterações ultra-estruturais observadas no figado dos ovinos desse experimento são semelhantes às descritas nas intoxicações experimentais por M. aeruginosa (Jackson et al. 1984) e C. laevigatum (Van Der Lugt et al. 1992) para esta espécie, em bovinos intoxicados experimentalmente por $C$. laevigatum (Van Der Lugt et al. 1991) e em ratos por microcistina-LR, princípio hepatotóxico de $M$. aeruginosa (Hooser et al. 1990). Há evidências de lesão endotelial caracterizada pela presença de restos celulares e de neutrófilos nos espaços de Disse associada à agregação plaquetária na área de dano endotelial. No entanto, não é possível determinar, tanto na microscopia óptica como ao microscópio eletrônico, se as lesões no endotélio dos sinusóides são primárias ou secundárias. Em estudos experimentais com a microcistina-LR em ratos (Hooser et al. 1990) e camundongos (Dabholkar \& Carmichael 1987), as conclusões sobre a localização primária da lesão são conflitantes. Em ratos é descrita como sendo nos hepatócitos e em camundongos como sendo nas células endoteliais dos sinusóides hepáticos.

As estruturas eosinofílicas observadas na microscopia óptica no citoplasma de hepatócitos, nos espaços de Disse e nos sinusóides e que não se coraram pela PTAH foram interpretadas como sendo citossegressomos ou desprendimento de fragmentos do citoplasma de hepatócitos para a luz dos sinusóides. As observações ultra-estruturais confirmam essa opinião. As lesões observadas ultra-estruturalmente nos hepatócitos são lesões clássicas e inespecíficas de degeneração e necrose celular (Slauson \& Cooper 1990).

Os macrófagos com citoplasma espumoso observados consistentemente nos linfonodos dos ovinos necropsiados não são provavelmente associados à ação de $X$. cavanillesii. Células de aspecto semelhante têm sido descritas no fígado, linfonodos hepáticos, mesentéricos e polpa esplênica de bovinos mantidos em pastagens de Brachiaria decumbens (Lemos et al. 1996). Desconhece-se a existência de pastos formados por essa forrageira na Região Sul do Brasil, mas a possibilidade de associação desses macrófagos à ingestão de alguma outra forrageira não pode ser descartada.

Agradecimentos.- Ao Srs. Wilson Fonseca e João Luiz Bastos, Proj. Sanidade Animal Embrapa/UFRRJ, pela preparação do material histológico e acerto da coloração. Ao Prof. David Driemeier, UFRGS, pela realização da coloração da PTAH. Ao Prof. Paulo Peixoto, UFRRJ, pelas correções e sugestões. Às Srtas. Silvia Grando e Denise Russowski, UFSM, pela preparação do material, fotos e diapositivos da microscopia eletrônica. À médica-veterinária Srta. Rosa Tatsch, Cachoeira do Sul, RS, pela visita à propriedade onde ocorreu o surto de intoxicação em bovinos.

\section{REFERÊNCIAS}

Calanasan C.A., Capon R.J., Gaul K.L., Klingenberg M., MacLeod J.K., Moeller P.D.R. \& Oelrichs P.B. 1985. Toxic analogues of wedeloside and carboxyatractyloside from Australian plant species, p. 238-244. In: Colegate S.M. \& Dorling P.R. (ed.) Plant-Associated Toxins. Short Run Press, Exeter.

Chaulet J.H.F., Peixoto P.V., Tokarnia C.H. 1990. Intoxicação experimental por Sessea brasiliensis (Solanaceae) em bovinos, ovinos e caprinos. Pesq. Vet. Bras. 10(3/4):71-84.

Cole R.J., Stuart B.P., Lansden J.A. \& Cox R.H. 1980. Isolation and redefinition of the toxic agent from cocklebur (Xanthium strumarium). J. Agric. Food Chem. 28(6):1330-1332.

Collazo L. \& Riet-Correa F. 1996. Experimental intoxication of sheep and cattle with Wedelia glauca. Vet. Hum. Toxicol. 38(3):200-203.

Dabholkar A.S. \& Carmichael W.W. 1987. Ultrastructural changes in the mouse liver induced by hepatotoxin from the freshwater cyanobacterium Microcystis aeruginosa strain 7820. Toxicon 25:285-292.

Driemeier D., Irigoyen L.F., Loretti A.P., Colodel E.M. \& Barros C.S.L. 1999. Intoxicação espontânea pelos frutos de Xanthium cavanillesii (Asteraceae) em bovinos no Rio Grande do Sul. Pesq. Vet. Bras. 19(1):12-18.

Dutra F., Riet-Correa F., Méndez M.C. \& Paiva N. 1997. Poisoning of cattle and sheep in Uruguay by sawfly (Perreya flavipes) larvae. Vet. Hum. Toxicol. 39(5):281-286.

Everist S.L. 1974. Poisonous Plants of Australia. Angus and Robertson Pty. Ltd, Sidney, p. 142-144. 
Gava A., Peixoto P.V. \& Tokarnia C.H. 1987. Intoxicação experimental por Vernonia molissima em ovinos e bovinos. Pesq. Vet. Bras. 7(2):33-41.

Gava A., Stolf L., Pilati C., Neves D.S. \& Viganó L. 1991. Intoxicação por Cestrum corymbosum var. hirsutum (Solanaceae) em bovinos no Estado de Santa Catarina. Pesq. Vet. Bras. 11(3/4):71-74.

Hooser S.B., Beasley V.R., Basgall E.J., Carmiachel W. W. \& Haschek W.M. 1990. Microcystin-LR-induced ultrastructural changes in rats. Vet. Path. 27:9-15.

Horváth Z. \& Modor V. 1970. [Xanthium italicum poisoning in piglets]. Magy. Allatorv. Lap. 25:349-354.

Jackson A.R.B., McInnes A., Falconer I.R. \& Runnegar M.T.C. 1984. Clinical and pathological changes in sheep experimentally poisoned by the bluegreen alga Microcystis aeruginosa. Vet. Pathol. 21:102-113.

Jones T.C., Hunt R.D. \& King N. 1997. Veterinary Pathology. 6th ed. Lea and Febiger, Philadelphia, p.1111-1147.

Kellerman T.S., Coetzer J.A.W. \& Naudé T.W. 1988. Plant Poisonings and Mycotoxicoses of Livestock in Southern Africa. Oxford University Press, Cape Town, p. 1-46.

Kenny G.C., Everist S.L. \& Sutherland A.K. 1950. Noogoora burr poisoning of cattle. Qld Agric. J. 70:172-177.

Kingsbury J.M. 1964. Poisonous Plants of the United States and Canada. Prentice-Hall, New Jersey, p 440-442.

Kissmann K. \& Groth D. 1992. Plantas Infestantes e Nocivas. Vol. 2. BASF, São Paulo, p. 389-397.

Lemos, R.A.A., Ferreira L.C.L., Silva S.M., Nakazato L. \& Salvador S.C. 1996. Fotossensibilização e colangiopatia associada a cristais em ovinos em pastagem com Brachiaria decumbens. Ciência Rural, Santa Maria, 26(1):109-113.

Marsh C.D., Glenwood C.R. \& Clawson A.B. 1924. Cockleburs (species of Xanthium) as poisonous plants. U. S. Dept. Agric.-Dept. Bull. 1274:1-24.

Martin T., Stair E.L. \& Dawson L. 1986. Cocklebur poisoning in cattle. J. Am. Vet. Med. Assoc. 189(5):562-563.

Martin T., Johson B.J., Sangiah S. \& Burrows G.E. 1992. Experimental cocklebur (Xanthium strumarium) intoxication in calves, p. 489-494. In: James L.F., Keeler R.F., Bailey E.M., Cheeke P.R. \& Hegarty M.P. (ed.) Proc. 3rd Int. Symposium on Poisonous Plants. Iowa University Press, Iowa.
Méndez M.C., Santos R.C. \& Riet-Correa F. 1994. Intoxicação por Xanthium sp. (carrapicho) em bovinos, p. 27-30. In: Schild, A.L., Riet-Correa F., Ferreira, J.L.M. \& Méndez M.C. (ed.) Boletim do Laboratório Regional de Diagnóstico. Documento 14. Editora e Gráfica Universitária, Pelotas.

Oelkers S. \& Oehme F. 1982. Cocklebur poisoning in swine. Bov. Pract. 3(2):1114.

Riet-Correa F., Schild A.L., Méndez, M.C., Oliveira, J.A., Gil-Turnes C. \& Gonçalves A. 1983. Atividades do Laboratório Regional de Diagnóstico e Doenças da Área de Influência no Período 1978-1982. Documento 4. Editora da Universidade Pelotas-RS, Pelotas, p. 87-88.

Riet-Correa F., Méndez M.C., Schild A.L., Meireles M.C.A. \& Scarsi R.M. 1984. Laboratório Regional de Diagnóstico - Doenças Diagnosticadas no Ano 1983. Documento 5. Editora da Universidade Pelotas, Pelotas, p. 32-33.

Santos R.C. 1997. Intoxicação experimental por Xanthium strumarium em bovinos e ovinos. M.Sc. Diss., Universidade Federal de Pelotas, Pelotas, RS. 32p.

Slauson D.O. \& Cooper B.J. 1990. Mechanisms of Disease. 2th ed. Williams \& Wilkins, Baltimore, p. 19-88.

Stuart B.P., Cole R.J. \& Gosser H.S. 1981. Cocklebur (Xanthium strumarium var. strumarium) intoxication in swine: review and redefinition of the toxic principle. Vet. Pathol. 18:368-383.

Van Der Lugt J.J., Nel P.W. \& Kitching J.P. 1991. The pathology of Cestrum laevigatum (Schletd.) poisoning in cattle. Onderstepoort J. Vet. Res. 58:211221.

Van Der Lugt J.J., Nel P.W. \& Kitching J.P. 1992. Experimentally-induced Cestrum laevigatum (Schletd.) poisoning in sheep. Onderstepoort J. Vet. Res. 59:135144.

Verstraete M., Vermylen J. \& Collen D. 1974, p. 447-455. Intravascular coagulation in liver disease. In: Creger W.P., Coggins C.H. \& Hancock E.W. (ed.) Ann. Rev. Med. 25

Witte S.T., Osweiler G.D., Stahr H.M. \& Mobley G. 1990. Cocklebur toxicosis in cattle associated with the consumption of Xanthium strumarium. J. Vet. Diagn. Invest. 2(4):263-267. 